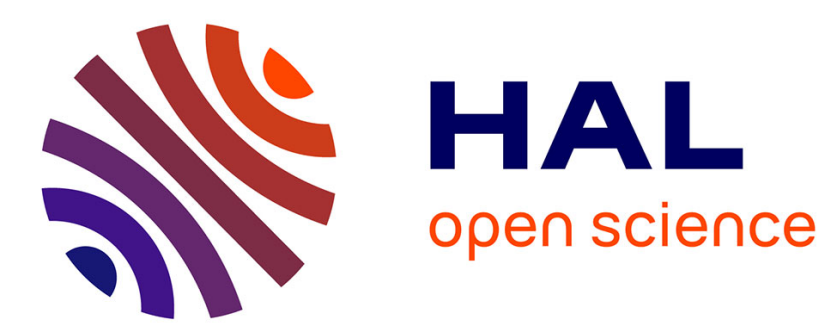

\title{
Interneuronal correlations dynamically adjust to task demands at multiple time-scales
}

S Ben Hadj Hassen, C Gaillard, E Astrand, C Wardak, S. Ben Hamed

\section{To cite this version:}

S Ben Hadj Hassen, C Gaillard, E Astrand, C Wardak, S. Ben Hamed. Interneuronal correlations dynamically adjust to task demands at multiple time-scales. 2020. hal-03045217

\section{HAL Id: hal-03045217 https://hal.science/hal-03045217}

Preprint submitted on 7 Dec 2020

HAL is a multi-disciplinary open access archive for the deposit and dissemination of scientific research documents, whether they are published or not. The documents may come from teaching and research institutions in France or abroad, or from public or private research centers.
L'archive ouverte pluridisciplinaire HAL, est destinée au dépôt et à la diffusion de documents scientifiques de niveau recherche, publiés ou non, émanant des établissements d'enseignement et de recherche français ou étrangers, des laboratoires publics ou privés. 


\title{
Interneuronal correlations dynamically adjust to task demands
}

at multiple time-scales

\author{
Ben Hadj Hassen ${ }^{1}$, S., Gaillard ${ }^{1}$, C., Astrand ${ }^{1,2}$, E., Wardak ${ }^{1,3}$, C., Ben Hamed ${ }^{1}$, S. \\ 1. Institut des Sciences Cognitives Marc Jeannerod, CNRS, UMR5229, 67 Boulevard Pinel, \\ 69675 Bron Cedex, France \\ 2. Mälardalen University, IDT, Högskoleplan 1, 72123 Västerås, Sweden \\ 3. Imagerie et Cerveau (iBrain), 10 Boulevard Tonnellé 37032 Tours Cedex 1, France \\ Lead contact: Suliann Ben Hamed, benhamed@isc.cnrs.fr \\ Corresponding author: Suliann Ben Hamed, benhamed@isc.cnrs.fr; Sameh Ben Hadj Hassen, \\ sameh.ben-hadj@isc.cnrs.fr
}

Key words: noise correlation, prefrontal cortex, macaque monkey, task difficulty, alpha oscillations, beta oscillations, cognitive demand, cognitive flexibility, rhythmic cognition,

\section{Summary}

Functional neuronal correlations between pairs of neurons are thought to play an important role in neuronal information processing and optimal neuronal computations during attention, perception, decision-making and learning. Here, we report dynamic changes in prefrontal neuronal noise correlations at multiple time-scales, as a function of task contingencies. Specifically, we record neuronal activity from the macaque frontal eye fields, a cortical region at the source of spatial attention top-down control, while the animals are engaged in tasks of varying cognitive demands. First, we show that noise correlations decrease as cognitive engagement and task demands increase, both across tasks and within-trials. Second, we demonstrate, for the first time, rhythmic modulations of noise correlations in the alpha and beta frequency ranges that account both for overt behavioral performance and for layer specific modulations in spike-field coherence. All this taken together demonstrates a strong functional role of noise correlations in cognitive flexibility. 


\section{Introduction}

Optimal behavior is the result of interactions between neurons both within and across brain areas. Identifying how these neuronal interactions flexibly adjust to the ongoing behavioral demand is key to understand the neuronal processes and computations underlying optimal behavior. Several studies have demonstrated that functional neuronal correlations between pairs of neurons, otherwise known as noise correlations, play an important role in perception and decision-making ${ }^{1-9}$. Specifically, several experimental and theoretical studies show that noise correlations have an impact on the amount of information that can be decoded for neuronal populations $s^{4,10-12}$ as well as on overt behavioral performance ${ }^{4,10-15}$. As a result, understanding how noise correlations dynamically adjust to task demands is a key step toward clarifying how neural circuits dynamically control information transfer, thereby optimizing behavioral performance.

Several sources of noise correlations have been proposed, arising from shared connectivity $^{16}$, global fluctuations in the excitability of cortical circuits ${ }^{17,18}$, feedback signals 19 or internal areal dynamics ${ }^{20-22}$, or bottom-up peripheral sensory processing ${ }^{23}$. From a cognitive point of view, noise correlations have been shown to change as a function of spatial attention $^{24}$, spatial memory ${ }^{25}$ and learning ${ }^{26,27}$, suggesting that they are subject both to rapid dynamic changes as well as to longer term changes, supporting optimal neuronal computations $^{27}$.

Here, we focus onto how multiple task contingencies induce dynamic changes in prefrontal neuronal noise correlations at multiple time-scales. Specifically, we record neuronal activity from the macaque frontal eye fields, a cortical region which has been shown to be at the source of spatial attention top-down control ${ }^{15,28-30}$ while the animals are engaged in tasks of varying cognitive demands, as assessed by their overt behavioral performance. Overall, we demonstrate that noise correlations dynamically adjust to the cognitive demand, decreasing as cognitive engagement and task demands increase. These dynamical changes take place both across task, as a function of task demands, and within trials, as a function of the probabilistic structure of the task, demonstrating a top-down control over this neuronal process. We also demonstrate, for the first time, rhythmic modulations of noise correlation in two specific functional frequency ranges: the alpha and beta frequency ranges. Crucially, these rhythmic modulations in noise correlations account both for overt behavioral performance and for layer specific modulations in spike-field coherence. All this taken together demonstrates a strong functional role of noise correlations in cognitive flexibility. 
62 These findings are discussed in relation with previously reported functional and structural 63 sources of variations in noise correlation and a comprehensive model of shared population neuronal variability is proposed.

Results

Our main goal in this work is to examine how the degree of cognitive engagement and task demands impact the neuronal population state as assessed from interneuronal noise correlations. Cognitive engagement was operationalized through tasks of increasing behavioral requirements. The easiest task (Fixation task, figure 1B.1) was a central fixation task in which monkeys were required to detect an unpredictable change in color of the fixation point, by producing a manual response within 150 to $800 \mathrm{~ms}$ from color change. The second task (Target detection task, figure 1B.2) added a spatial uncertainty on top of the temporal uncertainty of the event associated with the monkeys' response. This was a target detection task, in which the target could appear at one of four possible locations, at an unpredictable time from fixation onset. The monkeys had to respond to this target presentation by producing a manual response within 150 to $800 \mathrm{~ms}$ from color change. In the third task (Memory guided saccade task, figure 1B.3), monkeys were required to hold the position of a spatial cue in memory for 700 to $1900 \mathrm{~ms}$ and to perform a saccade towards that memorized spatial location on the presentation of a go signal. This latter task thus involved a temporal uncertainty but no spatial uncertainty. However, in contrast with the previous tasks, it required the production of a spatially oriented oculomotor response rather than a simple manual response. Accordingly, both monkeys had higher performances on the memory guided saccade task than on the target detection task (Figure 1C, Wilcoxon rank sum test, Monkey 1, $\mathrm{p}<0.01$, Monkey 2, $\mathrm{p}<0.05$ ), and higher performances on the target detection task than on the fixation task (Wilcoxon rank sum test, $\mathrm{p}<0.05$ ). 

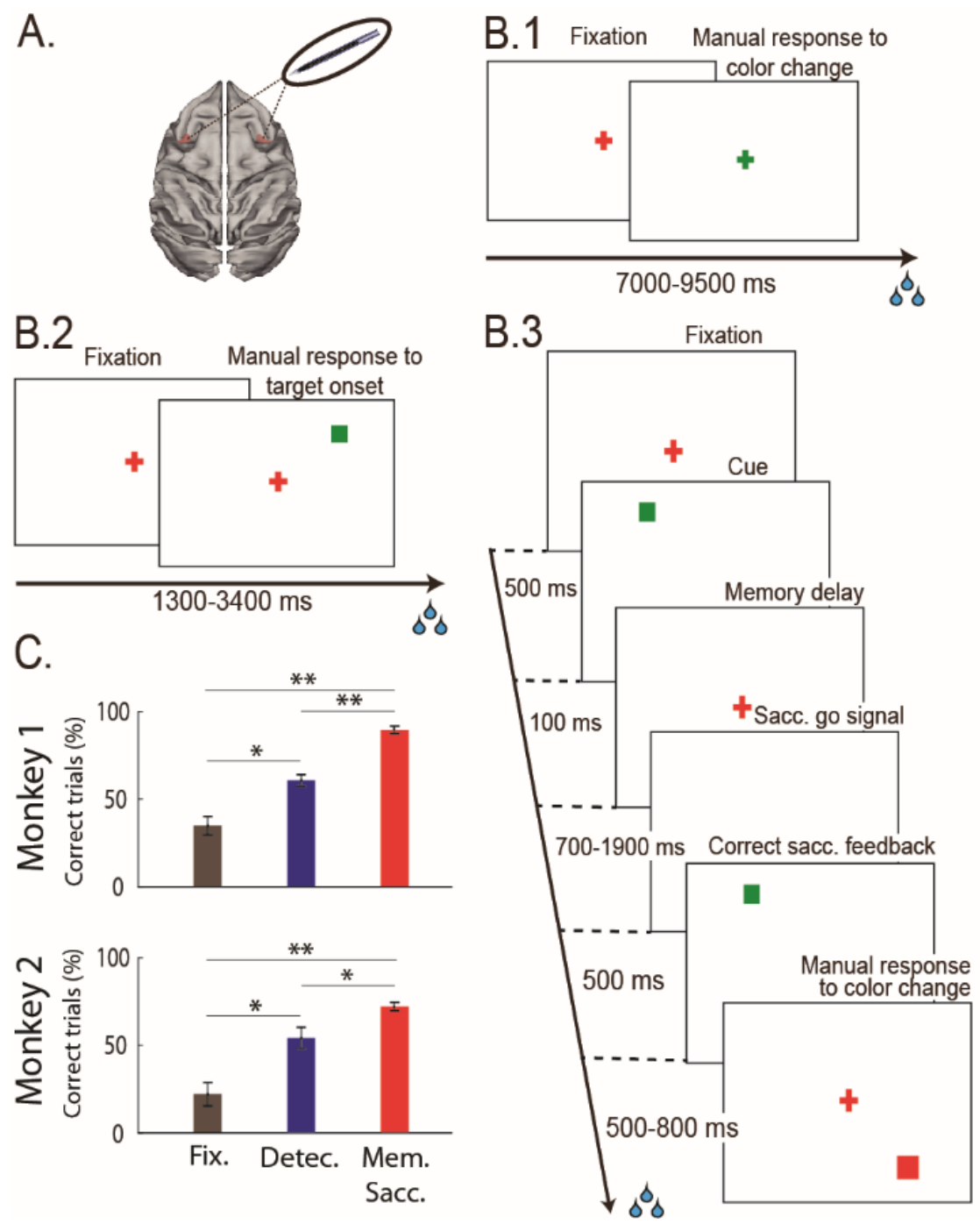

Figure 1: (A) Recordings sites. On each session, 24-contact recording probes were placed in the left and right FEFs. (B.1) Fixation task. Monkeys had to fixate a red central cross and were rewarded for producing a manual response $150 \mathrm{~ms}$ to $800 \mathrm{~ms}$ following fixation cross color change. (B.2) Target detection task. Monkeys had to fixate a red central cross and were rewarded for producing a manual response $150 \mathrm{~ms}$ to $800 \mathrm{~ms}$ from the onset of a low luminosity target at an unpredictable location out of four possible locations on the screen. (B.3) Memory-guided saccade task. Monkeys had to fixate a red central cross. A visual cue was briefly flashed in one of four possible locations on the screen. Monkeys were required to hold fixation until the fixation cross disappeared and then produce a saccade to the spatial location indicated by the cue within $300 \mathrm{~ms}$ from fixation point offset. On success, the cue reappeared and the monkeys had to fixate it. They were then rewarded for producing a manual response $150 \mathrm{~ms}$ to $800 \mathrm{~ms}$ following the color change of this new fixation stimulus. (C) Behavioral performance. Average percentage of correct trials across sessions for each tasks and each monkey with associated standard errors. 
Neuronal recordings were performed in the prefrontal cortex, specifically in the frontal eye field (FEF, figure 1A), a structure known to play a key role in covert spatial attention ${ }^{30-33}$. In each session, multi-unit activity (MUA) and local field potential (LFP) were recorded bilaterally, while monkeys performed these three tasks. In the following, the noise correlations between the different prefrontal signals of the same hemisphere were computed on equivalent task fixation epochs, away from both sensory intervening events and motor responses. In a first step, we analyzed how these noise correlations varied both across tasks, as a function of cognitive engagement and within-tasks, as a function of the probabilistic

increase. correlations. We relate these rhythmic variations to cognitive engagement and we show that they correlate with changes in the coupling between local field potentials and MUA spiking activity, in specific functional frequency bands.

\section{Noise correlations decrease as cognitive engagement and task requirements}

\section{increase.}

In order to characterize how inter-neuronal noise correlations vary as a function of cognitive engagement and task requirements, we proceeded as follows. In each session $(n=26)$, noise correlations were computed between each pair of task-responsive channels ( $n=671$, see Methods), over equivalent fixation task epochs, running from 300 to $500 \mathrm{~ms}$ after eye fixation onset. This epoch was at a distance from a possible visual or saccadic foveation response and in all three tasks, monkeys were requested to maintain fixation at this stage. It was also still early on in the trial, such that no intervening sensory event was to be expected by the monkey at this time. Importantly, fixation behavior, i.e. the distribution of eye position in within the fixation window, did not vary between the different tasks (Friedman test, $\mathrm{p}<0.001$ ). As a result, and because tasks were presented in blocks, any difference in noise correlations across tasks during this "neutral" fixation epoch are to be attributed to general non-specific task effects, i.e. differences in the degree of cognitive engagement and task demands. Noise correlations were significantly different between tasks (Figure 2A, ANOVA, $\mathrm{p}<0.001)$. Specifically, they were higher in the fixation task than in the target detection task (Figure 2A, Wilcoxon rank sum test, $\mathrm{p}<0.001$ ) and in the memory guided saccade task (Wilcoxon rank sum test, $\mathrm{p}<0.001$ ). They were also significantly higher in the target detection task than in the memory guided saccade task (Wilcoxon rank sum test, $\mathrm{p}<0.001$ ). Importantly, these significant changes in noise correlations existed in the absence of significant differences in mean firing rate (ANOVA, $>0.5$ ), standard error around this mean firing rate (ANOVA, 
$\mathrm{p}>0.6$ ), and Fano factor (ANOVA, $\mathrm{p}>0.7$, data not shwon). We thus describe that, in absence of any sensory or cognitive processing, noise correlations are strongly modulated by cognitive engagement and task demands.
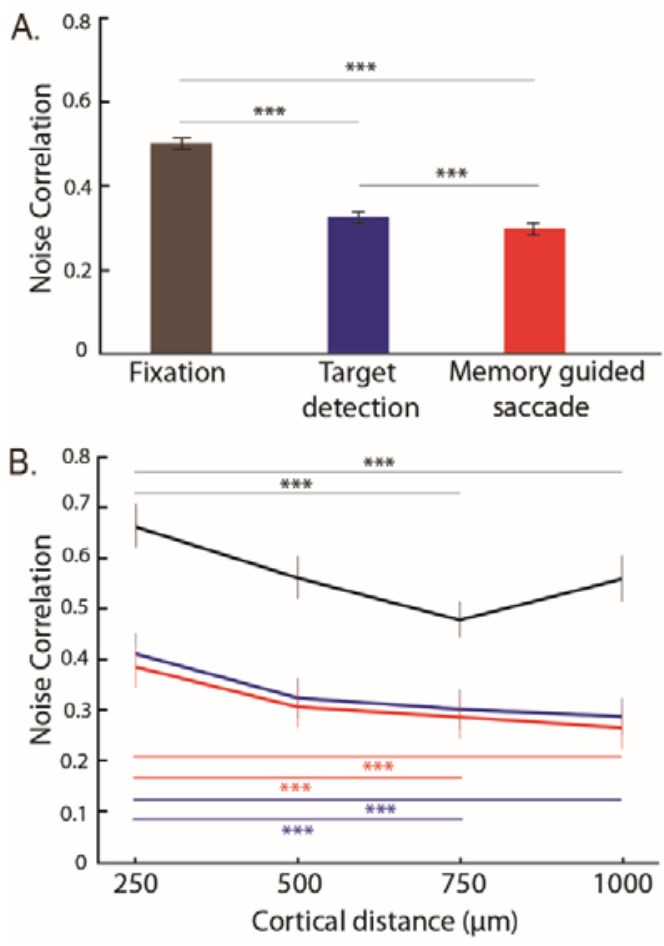

Figure 2: (A) Noise correlations as a function of task. Average noise correlations across sessions for each of the three tasks (mean +/- s.e., noise correlations calculated on the neuronal activities from 300 to 500 after eye fixation onset. Black: fixation task; blue: target detection task; red: memory guided saccade task. Stars indicate statistical significance following a one-way ANOVA; $*_{\mathrm{p}}<0.05 ; * *^{*}<0.01 ; * * *^{*}<0.001$. (B) Noise correlations as a function of cortical distance. Average noise correlations (mean +/- s.e.) across sessions, for each task (conventions as in (A)), from $300 \mathrm{~ms}$ to $500 \mathrm{~ms}$ after eye fixation onset, as a function of distance between pairs of channels: $250 \mu \mathrm{m} ; 500 \mu \mathrm{m} ; 750 \mu \mathrm{m} ; 1000 \mu \mathrm{m}$. Stars indicate statistical significance following a two-way ANOVA and ranksum post-hoc tests; ${ }^{*} \mathrm{p}<0.05$; $* * \mathrm{p}<0.01 ; * * * \mathrm{p}<0.001$. are task independent.

The task differences in noise correlations described above could reflect changes in the shared functional connectivity, within the large-scale parieto-frontal functional network the cortical region of interest belongs to ${ }^{16}$ or to global fluctuations in the excitability of cortical circuits $^{34,35}$. This large-scale hypothesis predicts that the observed changes in noise 
correlations are independent from intrinsic connectivity as assessed by the distance, the spatial selectivity or cortical layer between the pairs of signals across which noise correlations are computed. Alternatively, these task differences in noise correlations could reflect a more complex reweighing of functional connectivity and the excitatory/inhibitory balance in the area of interest, due to local changes in the random shared fluctuations in the pre-synaptic activity of cortical neurons ${ }^{4,16,36,37}$. This local hypothesis predicts that the observed changes in noise correlations depend onto intrinsic microscale connectivity. In the following, we characterize task differences in noise correlations as a function of cortical distance, spatial selectivity and cortical layer.

Cortical distance effects. Our recordings were performed as tangentially to FEF cortical surface as possible. The distance between the different recording probe contacts is thus a fair proxy to actual cortical tangential distance. Consistent with previous studies ${ }^{38-40}$, noise correlations significantly decreased as the distance between the pair of signals across which noise correlations were computed increased (Figure 2B). Importantly, this distance effect was present for all tasks and expressed independently of the main task effect described above (2-way ANOVA, Task x Distance, Task effect: $\mathrm{p}<0.001$; Distance effect: $\mathrm{p}<0.001$, interaction: $\mathrm{p}>0.05)$. Post-hoc analyses indicate that this distance effect is statistically significant, for all tasks, beyond $500 \mu \mathrm{m}$ (Wilcoxon rank sum test, Fixation task: $\mathrm{p}<0.001$ for a cortical distance of $750 \mu \mathrm{m}, \mathrm{p}<0.005$ for $1000 \mu \mathrm{m}$; Target detection task: $\mathrm{p}<0.001$ for 750 $\mu \mathrm{m}, \mathrm{p}<0.001$ for $1000 \mu \mathrm{m}$; Memory-guided saccade task: $\mathrm{p}<0.001$ for $750 \mu \mathrm{m}, \mathrm{p}<0.001$ for $1000 \mu \mathrm{m})$.

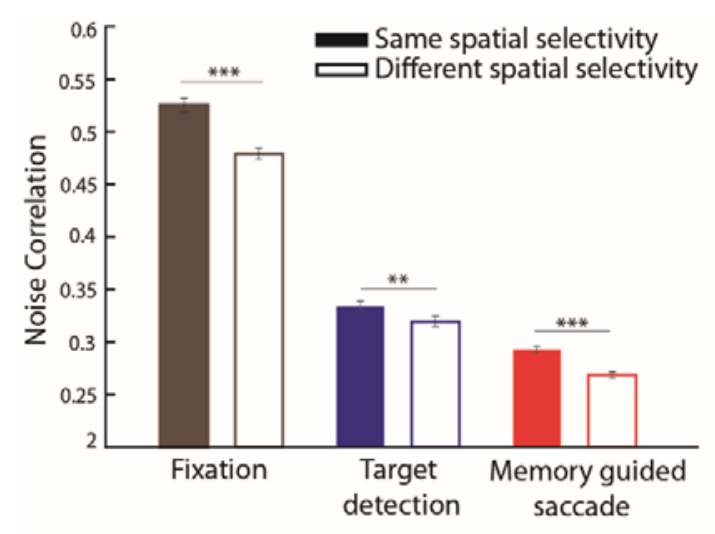

Figure 3: Noise correlations as a function of spatial selectivity. Average noise correlations (mean +/- s.e.) across sessions, for each tasks (conventions as in figure 2), from $300 \mathrm{~ms}$ to $500 \mathrm{~ms}$ after eye fixation onset, as a function of whether noise correlations are calculated over 
signals sharing the same spatial selectivity (full bars) or not (empty bars). Stars indicate statistical significance following a two-way ANOVA and ranksum post-hoc tests; $* \mathrm{p}<0.05$; $* * \mathrm{p}<0.01 ; * * * \mathrm{p}<0.001$.

Spatial selectivity effects. The spatial selectivity of each task-related MUA in response to cue presentation and saccade execution was assessed using an ANOVA (see methods). As described previously ${ }^{41,42}$, the receptive fields of FEF neurons are quite large and most MUA responded to cue presentation or saccade execution in more than one quadrant $(94 \%$ of MUA). For each MUA, we further identified the visual quadrant that elicited maximal neuronal response to cue or saccade execution, as well as, whenever possible the visual quadrant that didn't elicit any response. In the following, and under the assumption of a higher functional connectivity between pairs of MUA sharing the same spatial selectivity, we compared noise correlations between pairs of neurons sharing the same preferred quadrant and pairs for which the preferred quadrant of one MUA matched the unresponsive quadrant of the other MUA. Consistent with previous studies ${ }^{36}$, noise correlations were significantly lower for different spatial selectivity pairs than for same spatial selectivity pairs (Figure 3). This spatial selectivity effect was present for all tasks (2-way ANOVA, Task x Spatial selectivity, Task effect: $\mathrm{p}<0.001$; Spatial selectivity effect: $\mathrm{p}<0.001)$. Post-hoc analyses indicate that this spatial selectivity effect is statistically significant for all tasks (Wilcoxon rank sum test, Fixation task: $\mathrm{p}<0.001$; Target detection task: $\mathrm{p}<0.01$; Memory-guided saccade task: $\mathrm{p}<0.001$ ). However, spatial selectivity effects were not constant across tasks, possibly suggesting task-dependent functional changes in spatial selectivity based neuronal interactions (Task x Spatial selectivity interaction: $\mathrm{p}<0.05$ ).

Cortical layer effects. FEF neurons are characterized by a strong visual, saccadic, spatial memory and spatial attention selectivity ${ }^{30,42,43}$. Previous studies have shown that pure visual neurons are located in the input layers of the FEF while visuo-motor neurons are located in its output layers ${ }^{42,44-48}$. Independently, Buffalo et al. have shown that, in extrastriate area V4, the ratio between the alpha and gamma spike field coherence discriminated between LFP signals in deep (low alpha / gamma spike field coherence ratio) or superficial cortical layers (high alpha / gamma spike field coherence ratio) ${ }^{49}$. In our own data, because our recordings were performed tangentially to FEF cortical surface, we have no direct way of assigning the recorded MUAs to either superficial or deep cortical layers. However, the alpha / gamma spike field coherence ratio provides a very reliable segregation of visual and viso- 
216 motor MUAs (figure 4A). We thus consider that, as has been described for area V4, this

217 measure allows for a robust delineation of superficial and deep layers in area FEF. In the

218 following, we computed inter-neuronal noise correlations between three different categories

219 of pairs based on their assigned cortical layer: superficial/superficial pairs, superficial/deep 220 pairs and deep/deep pairs, where superficial MUA correspond to predominantly visual, low 221 alpha/gamma spike field coherence ratio signals and deep MUA correspond to predominantly 222 visuo-motor, high alpha/gamma spike field coherence ratio signals. Noise correlations varied 223 as a function of cortical layer (Figure 4B). This cortical layer effect was present for all tasks 224 and expressed independently of the main task effect described above (2-way ANOVA, Task $\mathrm{x}$ 225 Cortical layer, Task effect: $\mathrm{p}<0.001$; Cortical layer effect: $\mathrm{p}<0.001)$. As for spatial selectivity, 226 layer effects were not constant across tasks, possibly suggesting task-dependent functional 227 changes in within and across layer neuronal interactions (interaction: $\mathrm{p}<0.05$ ). Unexpectedly, 228 belonging to the same layer cortical layer didn't systematically maximize noise correlations. 229 Indeed, post-hoc analyses indicate significantly lower noise correlations between the 230 superficial/superficial pairs as compared to the deep/deep pairs (Wilcoxon rank sum test, 231 Fixation task: $\mathrm{p}<0.05$; Target detection task: $\mathrm{p}<0.05$; Memory-guided saccade task: $\mathrm{p}<0.01$ ). 232 Superficial/deep pairs sat in between these two categories and had significantly lower noise 233 correlations than the deep/deep pairs (Wilcoxon rank sum test, Fixation task: $\mathrm{p}<0.05$; Target 234 detection task: $\mathrm{p}<0.05$; Memory-guided saccade task: $\mathrm{p}<0.01)$ and higher noise correlations 235 than the superficial/superficial pairs, though this difference was never significant. 

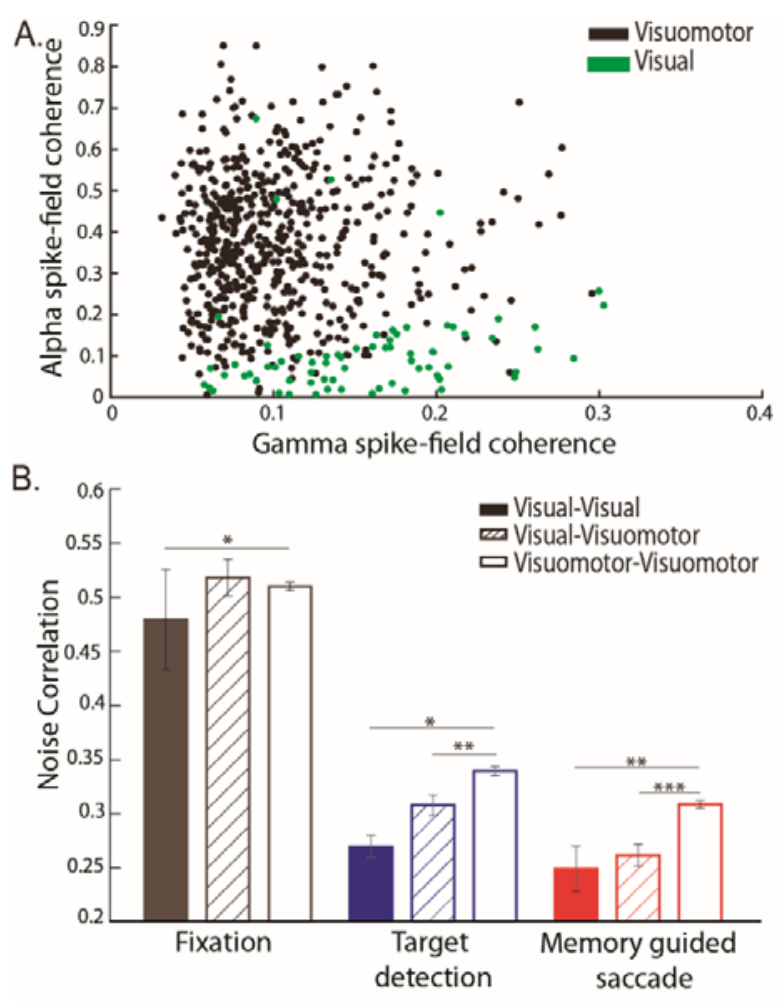

Figure 4: (A) Distribution of alpha spike-field coherence (6-16Hz) as a function of gamma $(40-60 H z)$ spike-field coherence for visual and visuomotor frontal eye field sites. Sites with visual selectivity but no motor selectivity (green, putative superficial sites) demonstrated stronger gamma-band spike-field coherence, whereas sites with visuomotor selectivity (black, putative deep sites) demonstrated stronger alpha-band spike-field coherence. (B) Noise correlations as a function of pair functional selectivity. Average of noise correlations (mean $+/$ - s.e.) across sessions, for each task (conventions as in figure 2), from $300 \mathrm{~ms}$ to $500 \mathrm{~ms}$ after eye fixation onset, as a function of pair functional selectivity: visual-visual, visualvisuomotor, visuomotor-visuomotor. Stars indicate statistical significance following a twoway ANOVA and ranksum post-hoc tests; *p<0.05; **p<0.01; ***p<0.001.

Overall, these observations support the co-existence of both a global large-scale change as well as a local change in functional connectivity. Indeed, task effects onto noise correlations build up onto cortical distance, spatial selectivity and cortical layer effects, indicating global fluctuations in the excitability of cortical circuits ${ }^{34,35}$. On top of this global effect, we also note more complex changes as reflected from statistical interactions between Task and spatial selectivity or layer attribution effects. This points towards more local changes in neuronal interactions, based on both 1) functional neuronal properties such as spatial selectivity that may change across tasks ${ }^{50-53}$ and 2) the functional reweighing of topdown and buttom-up processes ${ }^{28,30}$. 
Impact of the probabilistic structure of the task onto noise correlations.

Up to now, we have shown that noise correlations vary as a function of cognitive engagement and task demands. This suggests an adaptive mechanism that adjusts noise correlations to the ongoing behavior. On task shifts, this mechanism probably builds up during the early trials of the new task, past trial history affecting noise correlations in the current trials. $\operatorname{In}^{54}$ we show that, in a cued target detection task, while noise correlations are higher on miss trials than on hit trials, noise correlations are also higher on both hit and miss trials, when the previous trial was a miss as compared to when it was a hit. Here, one would expect that on the first trials of task shifts, noise correlations would be at an intermediate level between the previous and the ongoing task. Task shifts being extremely rare events in our experimental protocol, this cannot be confirmed. On top of this slow dynamics carry on effect, one can also expect faster dynamic adjustments to the probabilistic structure of the task. This is what we demonstrate below.

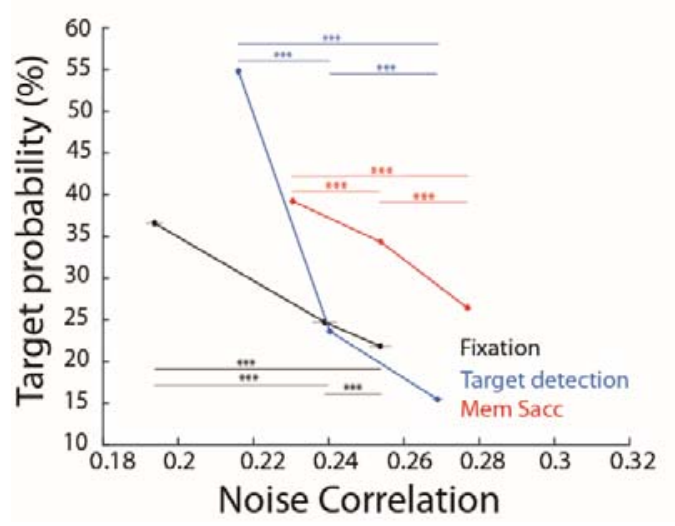

\section{Figure 5: Noise correlations decrease as function of expected response probability.} Average noise correlations (mean $+/$ - s.e.) across sessions, for each task (conventions as in figure 2), calculated on $200 \mathrm{~ms}$ before the target (Fixation and Target detection tasks) onset or saccade execution signal onset (memory guided saccade task), as a function of expected target probability. Each data point corresponds to noise correlations computed over trials of different fixation onset to event response intervals, i.e. over trials of different expected response probability. Stars indicate statistical significance following a two-way ANOVA and ranksum post-hoc tests; $* \mathrm{p}<0.05 ; * * \mathrm{p}<0.01 ; * * * \mathrm{p}<0.001$.

In each of the three tasks, target probability (saccade go signal probability in the case of the memory guided saccade task) varied as a function of time. As a result, early target onset trials had a different target probability than intermediate target onset trials than late target 
onset trials. Our prediction was that if monkeys had integrated the probabilistic structure of the task, this should reflect onto a dynamic adjustment of noise correlations as a function of target probability. Figure 5 confirms this prediction. Specifically, for all tasks, noise correlations were lowest in task epochs with highest target probability (Wilcoxon nonparametric test, $\mathrm{p}<0.001$ for all pair-wise comparisons). These variations between the highest and lowest target probability epochs were highly significant and in the order of the $15 \%$ or more (Fixation task: 15\%, Target detection task: 40\%, Memory-guided saccade task: 14\%). This variation range was lower than the general task effect we describe above but yet quite similar across tasks. Overall, this indicates that noise correlations are dynamically adjusted to the task structure, and are lowest at the time of highest behavioral demand in the trial.

\section{Rhythmic fluctuations in noise correlations.}

Up to now, we have described within and across task-related variations in noise correlations, building up onto intrinsic connectivity influences as reflected by cortical distance, spatial selectivity and layer attribution effects. Looking at noise correlations in time (figure 6A) reveals an additional source of variation, namely rhythmic changes in noise correlation levels, phase locked to fixation onset (Fixation and target detection task) or cue presentation (Memory guided saccade task). These rhythmic fluctuations take place in two distinct frequency ranges: a high alpha frequency range $(10-16 \mathrm{~Hz})$ and a beta frequency range $(20-30 \mathrm{~Hz})$, as quantified by a wavelet analysis (figure 6B). These oscillations can be described in all of the three tasks, this in spite of an overall higher background spectral power during the memory guided saccade task, both when noise correlations are calculated on trials in which spatial memory was instructed towards the preferred or the non-preferred location of the MUA signals (figure 6B, red and green curves respectively). Because spatial selective processes are at play in the memory guided saccade task, both for trials in which spatial memory is oriented towards the preferred MUA location (excitatory processes) or towards the non-preferred location (inhibitory processes), we will mostly focus on the fixation and the target detection tasks. When compensating the rhythmic modulations of noise correlations for background power levels (assuming an equal frequency power between all conditions beyond $30 \mathrm{~Hz}$ ), frequency power in the two ranges of interest are higher in the fixation task than in the target detection task (Friedman non-parametric test, all pairwise comparisons, $\mathrm{p}<0.001$ ), in agreement with the proposal that cognitive flexibility coincides with lower amplitude beta oscillations $^{55}$ and that attentional engagement coincides with lower amplitude alpha oscillations $^{56,57}$. Importantly, these oscillations are absent from the raw MUA signals 
316 (Friedman non-parametric test, all pairwise comparisons, $\mathrm{p}>0.2$ ), as well as when noise correlations are computed during the same task epochs but from neuronal activities aligned onto target presentation (or saccade go signal in the memory guided saccade task, Friedman non-parametric test, all pairwise comparisons, $\mathrm{p}>0.2$ ).

Importantly, in all of the three tasks, behavioral performance, defined as the proportion of correct trials as compared to error trials, varied as a function of alpha and beta noise correlation oscillations. Indeed, on a session by session basis, we could identify an optimal alpha $(10-16 \mathrm{~Hz})$ phase for which the behavioral performance was maximized, in antiphase with a bad alpha phase, for which the behavioral performance was lowest (figure 6C). These effects were highest in the fixation task (34.6\% variation in behavioral performance) and lowest though significant in the memory-guided saccade task $(13.3 \%$ in the target detection task and $9.5 \%$ in the memory guided saccade task). Similarly, an optimal beta $(20-30 \mathrm{~Hz})$ phase was also found to modulate behavioral performance in the same range as the observed alpha behavioral modulations $(28.3 \%$ variation in behavioral performance in the fixation task, $19.2 \%$ in the target detection task and $11 \%$ in the memory guided saccade task). As a result, Alpha and beta oscillation phase in noise correlations were predictive of behavioral performance, and the strength of these effects co-varied with alpha and beta oscillation amplitude in noise correlations, being higher in the fixation task, than in the target detection task than in the memory guided saccade task.
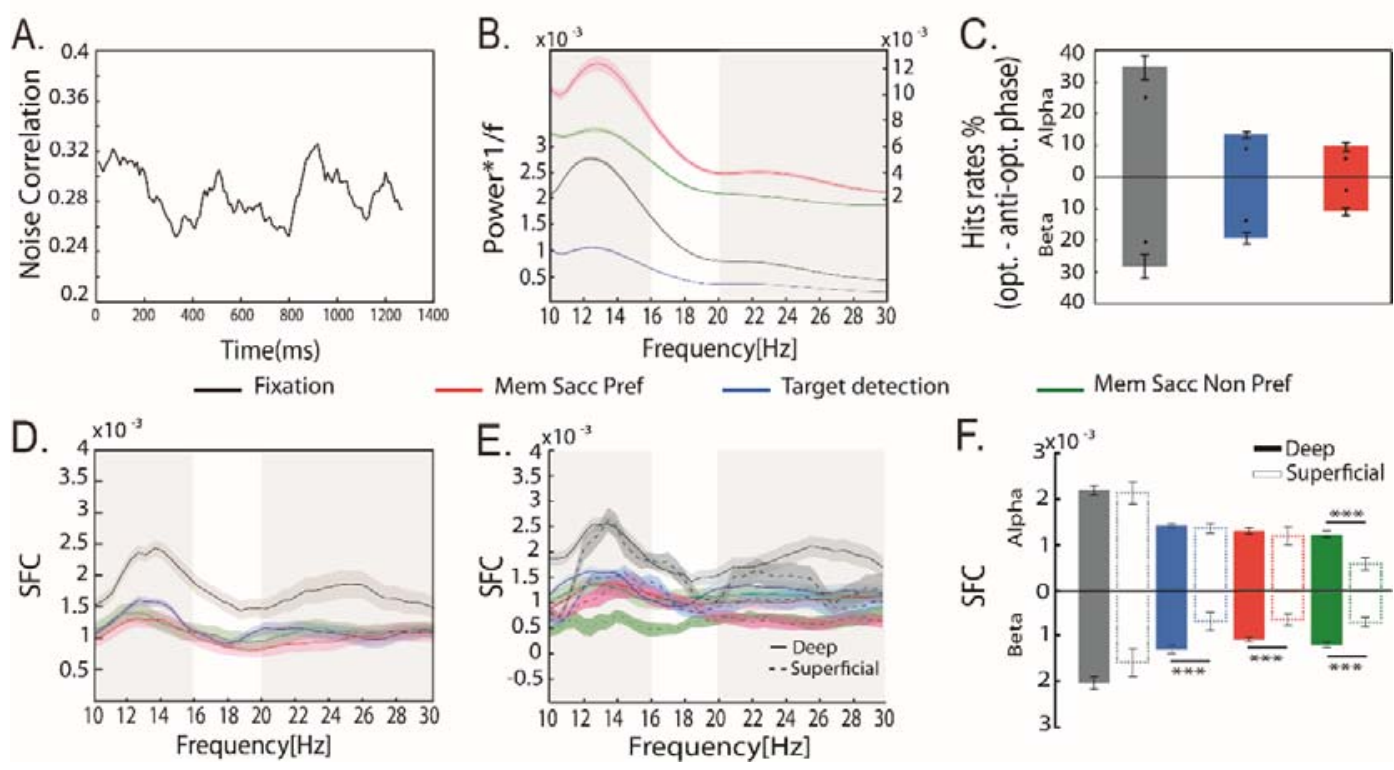

Figure 6: Rhythmic fluctuations in noise correlations modulate behavioral response and spike-field coherence in upper input cortical layers. (A) Single memory guided saccade 
session example of noise correlation variations as a function of trial time. (B) $1 / \mathrm{f}$ weighted power frequency spectra of noise correlation in time (average +/- s.e.m), for each task, calculated from $300 \mathrm{~ms}$ to $1500 \mathrm{~ms}$ from fixation onset (Fixation and Target detection tasks) or following cue offset (Memory guided saccade task). (C) Hit rate modulation by alpha (top histogram) and beta (bottom histogram) noise correlation at optimal phase as compared to anti-optimal phase for all three tasks (color as in (B), average $+/$ - s.e., dots represent the $95 \%$ confidence interval under the assumption of absence of behavioral performance phase dependence). (D) Spike field coherence between LFP and spike data as a function of frequency, time intervals as in (B). (E) Spike field coherence calculated as in (C) but as a function of the layer attribution of each signal, time intervals as in (B). (F) Average SFC (+/s.e.) in alpha $(10-16 \mathrm{~Hz}$, top histogram) and beta $(20-30 \mathrm{~Hz}$, bottom histogram) for each task and both of superficial and deep cortical layer signals (t-test, $* * *$ : $p<0.001$ ).

High alpha and beta oscillations in the local field potentials (LFP) are ubiquitous and are considered to reflect long-range processes. Beta oscillations have been associated with cognitive control and cognitive flexibility. On the other hand, alpha oscillations are associated with attention, anticipation ${ }^{56,57}$, perception ${ }^{58-60}$, and working memory ${ }^{61}$. We hypothesized a functional link between these LFP oscillations and the rhythmic oscillatory pattern of noise correlations. Figure 6D represents spike field coherence (SFC) between spiking activity and LFP signals (see Materials and Methods) computed during a $1200 \mathrm{~ms}$ time interval starting $300 \mathrm{~ms}$ after either fixation onset (Fixation and Target detection task) or cue offset (Memory guided saccade task). SFC peaks at both the frequency ranges identified in the noise correlation spectra, namely the high alpha range $(10-16 \mathrm{~Hz})$ and the beta range $(20-30 \mathrm{~Hz})$. Importantly, this SFC modulation is highest for the fixation task as compared to the target detection task, thus matching the oscillatory power differences observed in the noise correlations. SFC are lowest in the memory guided saccade task whether considering preferred or non-preferred spatial processing. This is probably due to the fact that the cue to go signal interval of the memory guided saccade task involves memory processes that are expected to desynchronize spiking activity with respect to the LFP frequencies of interest ${ }^{49}$. This will need to be further explored.

In figure 4, we show layer specific effects onto noise correlations that build up onto the global task effects. An important question is whether these layer effects result from layer specific changes in SFC. Figure $6 \mathrm{E}$ represents the SFC data of figure $6 \mathrm{D}$, segregated on the bases of the attribution of the MUA to either superficial or deep cortical FEF layers. While SFC modulations are observed in the same frequencies of interest as in figure $6 \mathrm{D}$, clear layer 
specific differences can be observed (figure 6F). Specifically, beta range SFC are markedly significantly lower in the superficial layers than in the deep layers, for both the detection task and the memory guided saccade task. This points towards a selective control of correlated noise in input, superficial FEF layers. In contrast, alpha range SFC are significantly lower in the superficial layers than in the deep layers only in the memory guided saccade, and specifically when spatial memory is oriented towards a non-preferred location. This points towards overall weaker layer differences for alpha SFC. Alternatively, alpha SFC could result from a different mechanism than beta SFC. This will need to be further explored. Thus in spite of the fact that a comprehensive layer effect of alpha SFC is still lacking at this stage, both alpha and beta noise correlation rhythmicity co-vary with 1) selective SFC modulations in the alpha and beta frequency ranges (these latter being more pronounced in the superficial input cortical layers than in the deeper cortical layers) as well as with 2) pronounced variations in overt behavioral performance.

Overall, we thus identify a last functional oscillatory source of variations in noise correlations in the alpha and beta ranges that both have an important functional relevance, as they coincide with systematic variations in behavioral performance. These oscillations reflect selective changes in SFC, more pronounced in the superficial than in the deep cortical layers. This oscillatory source of variation in noise correlations adds up on top of the previously identified sources of variation, namely global task demands and the probabilistic structure of the task.

\section{Discussion}

In this work, our main goal was to examine the impact of cognitive engagement and task demands onto the neuronal population shared variability as assessed from interneuronal noise correlations at multiple time scales. Recordings were performed in the macaque frontal eye fields, a cortical region in which neuronal noise correlations have been shown to vary as a function of spatial attention ${ }^{24}$ and spatial memory ${ }^{25,62}$. Noise correlations were computed over equivalent behavioral task epochs, prior to response production, during a delay in which eyes were fixed and in the absence of any intervening sensory event or motor response. As a result, any observed differences in noise correlations are to be assigned to an endogenous source of shared neuronal variability. 
Overall, we demonstrate, for the first time, that noise correlations dynamically adjust

406

407

408

409

410

411

412

413

414

415

416

417

418

419

420

421

422

423

424

425

426

427

428

429

430

431

432

433

434

435

436

437

438 to task demands at different time scales. Specifically, we show that noise correlations decrease as cognitive engagement and task demands increase. These task-related variations in noise correlations co-exist with within-trial dynamic changes related to the probabilistic structure of the tasks as well as with long- and short-range oscillatory brain mechanisms. These findings are discussed below in relation with previously reported functional and structural sources of variations in noise correlation and a comprehensive model of shared population neuronal variability is proposed.

\section{Shared neuronal population response variability dynamically adjusts to the behavioral demands.}

Noise correlations have been shown to vary with learning or changes in behavioral state $\left(\mathrm{V} 1^{40,63-65}, \mathrm{~V}^{24,66-68}\right.$ and $\left.\mathrm{MT}^{4,69,70}\right)$. For example, shared neuronal population response variability was lower in V1 in trained than in naïve monkeys ${ }^{26}$. More recently, Ni et al. describe, within visual areas, a robust relationship between correlated variability and perceptual performance, whether changes in performance happened rapidly (attention instructed by a spatial cue) or slowly (learning). This relationship was robust even when the main effects of attention and learning were accounted for $^{27}$. Here, we question whether changes in noise correlations can be observed simultaneously at multiple time scales. We describe two different times scales at which noise correlations dynamically adjust to the task demands.

The first adjustment in noise correlations we describe is between tasks, that is between blocked contexts of varying cognitive demand, the monkeys knowing that general task requirements will be constant over a hundred of trials or more. Task performance is taken as a proxy to cognitive adjustment to the task demands and negatively correlates with noise correlations in the recorded population. Shared neuronal population variability measure is largest in the fixation task as compared to the two other tasks, by almost $30 \%$. The difference between noise correlations in the target detection task as compared to the guided memory saccade task is in the range of $2 \%$, closer to what has been previously reported in the context of noise correlation changes under spatial attention ${ }^{24}$ or spatial memory manipulations. Importantly, these changes in noise correlations are observed in the absence of significant variations in individual neuronal spiking statistics (average spiking rates, spiking variability or associated Fano factor). To our knowledge, this is the first time that such task effects are described onto noise correlations. This variation in noise correlations as a function of cognitive engagement and task requirements suggests an adaptive mechanism that adjusts 
noise correlations to the ongoing behavior. Such a mechanism is expected to express itself at different timescales, ranging from the task level, to the across trial level to the within trial level. This is explored next.

It is unclear whether the transitions between high and low noise correlation states when changing from one task to another are fast (over one or two trials) or slow (over tens of trials). $\mathrm{In}^{54}$, we show that noise correlations vary as a function of immediate trial past history. Specifically, noise correlations are significantly higher on error trials than on correct trials, both measures being higher if the previous trial is an error trial than if the previous trial is a correct trial. We thus predict a similar past history effect to be observed on noise correlations at transitions between tasks, and we expect for example, noise correlations to be lower in fixation trials that are preceded by a target detection trial, than in trials preceded by fixation trials. In our experimental design, task transitions are unfortunately rare events, precluding the computation of noise correlations on these transitions.

However, our experimental design affords an analysis at a much finer timescale, i.e. the description of a dynamical adjustment in noise correlations within trials. Specifically, we show that noise correlations dynamically adjust to the probability of occurrence of a behaviorally key task event associated with the reward response production (target presentation on the fixation and target detection tasks or saccade go signal on the memory guided saccade task). In other words, shared neuronal population response variability dynamically adjusts to higher demand task epochs. As expected from the general idea that low noise correlations allow for optimal signal processing ${ }^{12,71,72}$, we show that, on each of the three tasks, at any given time in the fixation epoch prior to response production, the higher the probability of having to initiate a response, the lower the noise correlations.

Overall, this supports the idea that noise correlations is a flexible physiological parameter that dynamically adjusts at multiple timescales to optimally meet ongoing behavioral demands, as has been demonstrated in multisensory integration ${ }^{73}$ and through learning and attention ${ }^{27}$. The mechanisms through which this possibly takes place are discussed below.

\section{Long-range and short-range mechanisms for noise correlation dynamics.}

As described by previous studies, in all the three tasks, interneuronal noise correlations significantly decay as a function of cortical distance ${ }^{40,74,75}$. Likewise, in all the three tasks, noise correlations are significantly higher among neurons sharing the same spatial selectivity as compared to between neurons with different spatial selectivity ${ }^{4,13,36,40,69,71,76}$, supporting a 
472 functional role for noise correlations ${ }^{7}$ in the framework of biased competition models of 473 perception ${ }^{77}$. Last, in all three tasks, noise correlations depend on the functional selectivity of 474 the neurons. Indeed, noise correlations were lowest for visual MUA pairs, highest for 475 visuomotor MUA pairs and intermediate for visuo-visuomotor MUA pairs layers ${ }^{42,44-48}$. This thus points towards local layer specific noise correlation mechanisms.

Noise correlations are thought to vary due to global fluctuations in the excitability of cortical circuits at large ${ }^{34,35}$ as well as to fluctuations specific to a given functional network ${ }^{16}$. Alternatively, variations in shared neuronal population response variability are also proposed to result from changes in local processes, due to a reweighing of local functional connectivity, local excitatory/inhibitory balance and/or a change in the random shared fluctuations in the pre-synaptic activity of cortical neurons ${ }^{4,16,36,37}$. These two hypotheses are not mutually exclusive. The question is whether the task demand effects we describe here affect noise correlations irrespective of cortical distance, neuronal spatial selectivity and functional/layer specificity, or whether an interaction can be identified between task demand effects and cortical distance, neuronal spatial selectivity and functional/layer specificity. An absence of interactions would point towards a global noise correlation modulatory mechanism while an interaction would point towards more local noise correlation modulatory mechanism.

Our observations support the co-existence of both long-range global mechanisms and short-range local mechanisms. Indeed, we identify a very clear scaling of cortical distance, neuronal spatial selectivity and functional/layer specificity effects by general task demand, reflecting global influences onto noise correlations. On top of these global effects, we also note more complex changes in noise correlations that point towards local changes in neuronal interactions. Indeed, while task demand modulates noise correlations independently of cortical distance effects, we describe statistical interactions between task demand effects and neuronal spatial selectivity and functional/layer specificity effects. Specifically, neuronal spatial selectivity effects are more pronounced in the less demanding fixation task, than in the more demanding target detection and memory-guided saccade tasks. This suggests an active mechanism whereby noise correlations across neurons sharing the same spatial selectivity are selectively decreased under task demand, irrespectively of changes in noise correlations in the neurons of different spatial selectivity. Alternatively these selective changes in noise correlation can result from task-related dynamic changes in the neuronal spatial selectivity ${ }^{50-}$ 
505 This suggests an active mechanism whereby noise correlations across visual neuronal pairs

506 (and to a lesser degree visuo-visuomotor neuronal pairs) are selectively decreased under task 507 demand, irrespectively of changes in noise correlations in the visuomotor neuronal pairs, 508 possibly relying on a dynamic functional reweighing of top-down and buttom-up processes $509 \quad 28,30$.

510 All this taken together indicates that changes in noise correlations in the FEF as a 511 function of task demand both depend onto long-range global mechanisms and short-range 512 functional and layer specific mechanisms.

\section{Rhythmic fluctuations in noise correlations.}

In the above, we describe changes in noise correlations between tasks as a function of the cognitive demand, as well as within trials, as a function of the probabilistic structure of each task. In addition to these task-related dynamics, we also observe rhythmic fluctuations in noise correlations. These fluctuations are clearly identified in the high alpha frequency range $(10-16 \mathrm{~Hz})$ and to a lesser extent in the low gamma frequency range $(20-30 \mathrm{~Hz})$. To our knowledge, this is the first time that such rhythmic variations in noise correlations are reported. The question is whether these oscillations have a functional relevance or not.

From a behavioral point of view, we show that overt behavioral performance in the three tasks co-vary with both the $10-16 \mathrm{~Hz}$ and $20-30 \mathrm{~Hz}$ noise correlation oscillations. In other words, these oscillations account for more than $10 \%$ of the behavioral response variability, strongly supporting a functional role for these alpha and beta oscillations.

From a functional point of view, attention directed to the receptive field of neurons has been shown to both reduce noise correlations ${ }^{24}$ and spike-field coherence in the gamma range

$527\left(\mathrm{~V}^{78}\right.$, it is however to be noted that Engel et al. describe increased spike-field coherence in $528 \mathrm{~V} 1$, the gamma range under the same conditions, hinting towards areal specific differences ${ }^{79}$ ). 529 In our hands, the rhythmic fluctuations in noise correlations co-exist with increased spike530 field coherence in the very same $10-16 \mathrm{~Hz}$ and $20-30 \mathrm{~Hz}$ frequency ranges we identify in the 531 noise correlations. This suggests that changes in shared neuronal variability possibly arise 532 from changes in the local coupling between neuronal spiking activity and local field 533 potentials. Supporting such a functional coupling, both the rhythmic fluctuations in noise 534 correlations and spike-field coherence in the frequencies of interest are highest in the fixation 535 task as compared to the other two tasks. 
Beta oscillations in the local field potentials (LFP) are considered to reflect long-range 537 processes and have been associated with cognitive control and flexibility ${ }^{28,55,79-82}$ as well as 538 with motor control ${ }^{83-85}$ ( for review $\mathrm{se}^{55}$ ). Specifically, lower beta power LFPs has been 539 associated with states of higher cognitive flexibility. In our hands, lower beta in noise 540 correlations correspond to higher cognitive demands. We thus hypothesize a functional link 541 between these two measures, LFP oscillations locally changing spiking statistics, i.e. noise 542 correlations, by a specific spike-field coupling in this frequency range. Supporting a long543 range origin of these local processes (figure 7, inset), we show that spike-field coherence in 544 this beta range strongly decreases in the more superficial cortical layers as compared to the 545 deeper layers, as task cognitive demand increases. On the other hand, alpha oscillations are 546 associated with attention, anticipation ${ }^{56,57}$, perception ${ }^{58-60}$, and working memory ${ }^{61}$. As for beta 547 oscillations, lower alpha in noise correlations, and accordingly in spike-field coherence, 548 correspond to higher cognitive demands. In contrast with what is observed for beta spike-field 549 coherence, alpha spike-field coherence does not exhibit any layer specificity across task 550 demands. Thus overall, alpha and beta rhythmicity account for strong fluctuations in 551 behavioral performance, as well as for changes in spike-field coherence. However, beta 552 processes seem to play a distinct functional role as compared to the alpha processes, as their 553 effect is more marked in the superficial than in the deeper cortical layers. These observations 554 coincide with recent evidence that cognition is rhythmic ${ }^{86,87}$ and that noise correlations play a 555 key role in optimizing behavior to the ongoing time-varying cognitive demands ${ }^{27}$. 


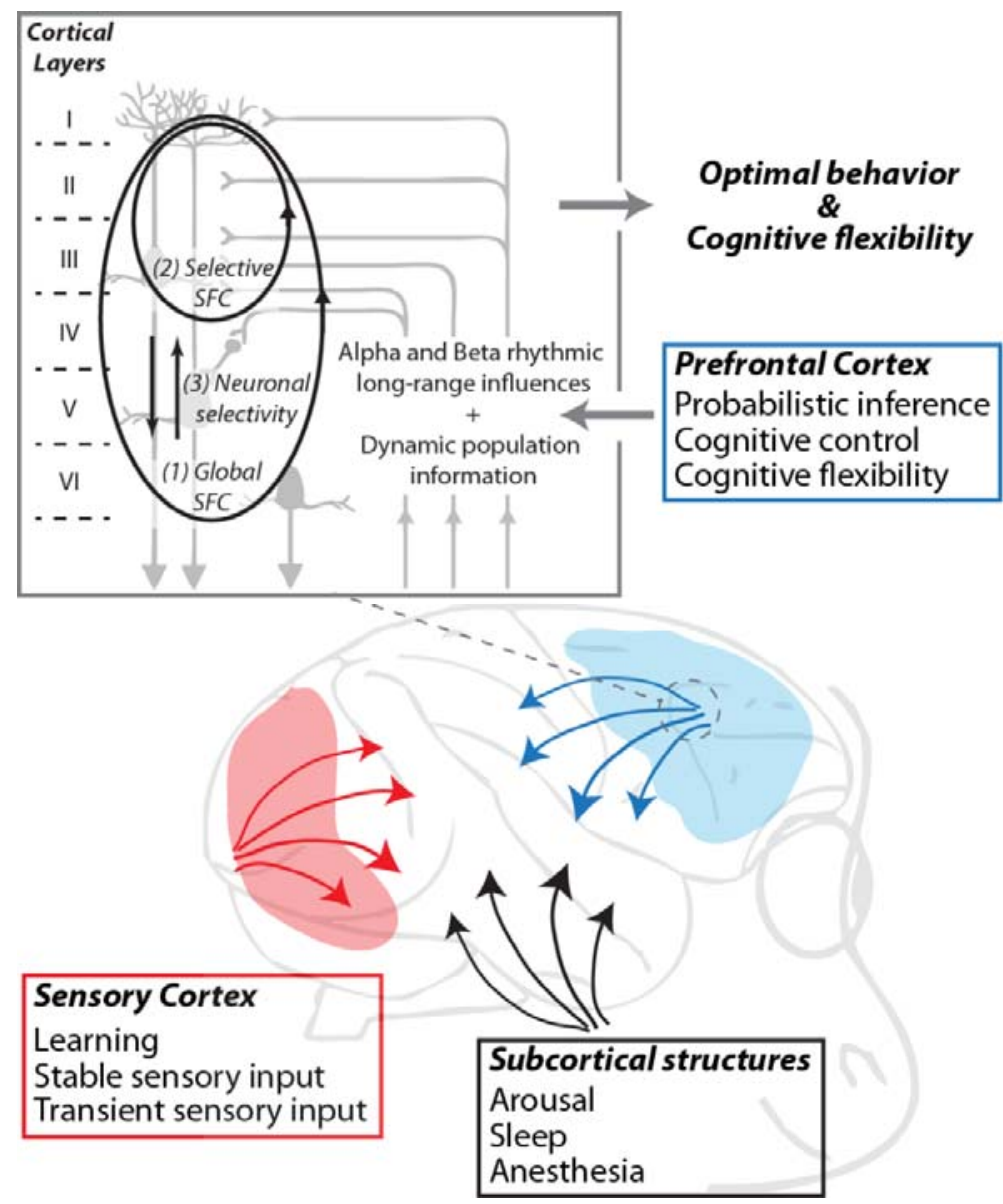

Figure 7: Schematic representation of local and long-range mechanisms for the dynamic and flexible adjustment of noise correlations to ongoing task demands.

\section{Conclusion.}

We thus demonstrate that noise correlations are highly dynamic, adjusting to the ongoing behavioral demands, both across tasks and within trials. They are also rhythmic, time varying in the alpha and beta frequency ranges. These rhythmic changes account both for overt behavioral performance as well as for selective changes in spike-field coupling in prefrontal superficial input cortical layers.

These dynamic adjustments in noise correlations correspond to a top-down control (Figure 7, blue) over local neuronal processes, mediated through long-range inter-areal influences. Alpha and beta rhythmicity appear to play a major role in this process, beta rhythmicity being involved in a selective superficial SFC modulation (Figure 7, inset, (2)), and alpha rhythmicity being involved in a more global SFC modulation (Figure 7, inset, (1)). These rhythmic processes co-exist with selective changes in noise correlations as a function of 
573 neuronal selectivity (Figure 7, inset, (3)). These top-down dynamic adjustments in noise

574 correlations are expected to add up onto state-related changes in noise correlations (Figure 7,

575 black), possibly mediated through neuromodulatory mechanisms, and sensory bottom-up

576 induced changes in noise correlations (Figure 7, red).

577 Overall, neuronal correlations are to be considered as a key neuronal mechanism 578 through which top-down and bottom-up neuronal influences are integrated to optimize 579 behavioral performance, along the same integrative rules as described for other neuronal 580 activity statistics.

581 
582

583

584

585

586

587

588

589

590

591

592

593

594

595

596

597

598

599

600

601

602

603

604

605

606

607

608

609

610

611

\section{Material and methods}

\section{Ethical statement}

All procedures were in compliance with the guidelines of European Community on animal care (Directive 2010/63/UE of the European Parliament and the Council of 22 September 2010 on the protection of animals used for scientific purposes) and authorized by the French Committee on the Ethics of Experiments in Animals (C2EA) CELYNE registered at the national level as C2EA number 42 (protocole C2EA42-13-02-0401-01).

\section{Surgical procedure:}

As in $^{54}$, two male rhesus monkeys (Macaca mulatta) weighing between 6-8 kg underwent a unique surgery during which they were implanted with two MRI compatible PEEK recording chambers placed over the left and the right FEF hemispheres respectively (figure 1a), as well as a head fixation post. Gas anesthesia was carried out using Vet-Flurane, 0.5 - 2\% (Isofluranum 100\%) following an induction with Zolétil 100 (Tiletamine at $50 \mathrm{mg} / \mathrm{ml}, 15 \mathrm{mg} / \mathrm{kg}$ and Zolazepam, at $50 \mathrm{mg} / \mathrm{ml}, 15 \mathrm{mg} / \mathrm{kg}$ ). Post-surgery pain was controlled with a morphine pain-killer (Buprecare, buprenorphine at $0.3 \mathrm{mg} / \mathrm{ml}, 0.01 \mathrm{mg} / \mathrm{kg}$ ), 3 injections at 6 hours interval (first injection at the beginning of the surgery) and a full antibiotic coverage was provided with Baytril 5\% (a long action large spectrum antibiotic, Enrofloxacin $0.5 \mathrm{mg} / \mathrm{ml}$ ) at $2.5 \mathrm{mg} / \mathrm{kg}$, one injection during the surgery and thereafter one each day during 10 days. A $0.6 \mathrm{~mm}$ isomorphic anatomical MRI scan was acquired post surgically on a $1.5 \mathrm{~T}$ Siemens Sonata MRI scanner, while a high-contrast oil filled grid (mesh of holes at a resolution of $1 \mathrm{mmx} 1 \mathrm{~mm}$ ) was placed in each recording chamber, in the same orientation as the final recording grid. This allowed a precise localization of the arcuate sulcus and surrounding gray matter underneath each of the recording chambers. The FEF was defined as the anterior bank of the arcuate sulcus and we specifically targeted those sites in which a significant visual and/or oculomotor activity was observed during a memory guided saccade task at 10 to $15^{\circ}$ of eccentricity from the fixation point (figure $\left.1 \mathrm{~A}\right)$. In order to maximize taskrelated neuronal information at each of the 24-contacts of the recording probes, we only recorded from sites with task-related activity observed continuously over at least $3 \mathrm{~mm}$ of depth.

\section{Behavioral task:}


612 During a given experimental session, the monkeys were placed in front of a computer 613 screen $(1920 \times 1200$ pixels and a refresh rate of $60 \mathrm{~Hz})$ with their head fixed. Their water 614 intake was controlled so that their initial daily intake was covered by their performance in the 615 task, on a trial by trial basis. This quantity was complemented as follows. On good 616 performance sessions, monkeys received fruit and water complements. On bad performance 617 sessions, water complements were provided at a distance from the end of the session. Each 618 recording session consisted of random alternations of three different tasks (see below and 619 figure $1 \mathrm{~b}$ ), so as to control for possible time in the session or task order effects. For all tasks, 620 to initiate a trial, the monkeys had to hold a bar in front of the animal chair, thus interrupting 621 an infrared beam. (1) Fixation Task (figure 1B.1): A red fixation cross $\left(0.7 \mathrm{x} 0.7^{\circ}\right)$, appeared 622 in the center of the screen and the monkeys were required to hold fixation during a variable 623 interval randomly ranging between 7000 and $9500 \mathrm{~ms}$, within a fixation window of $1.5 \times 1.5^{\circ}$, 624 until the color change of the central cross. At this time, the monkeys had to release the bar 625 within 150-800 ms after color change. Success conditioned reward delivery. (2) Target 626 detection Task (figure 1B.2): A red fixation cross $\left(0.7 \times 0.7^{\circ}\right)$, appeared in the center of the 627 screen and the monkeys were required to hold fixation during a variable interval ranging 628 between 1300 and $3400 \mathrm{~ms}$, within a fixation window of $1.5 \times 1.5^{\circ}$, until a green squared target $629\left(0.28 \times 0.28^{\circ}\right)$ was presented for $100 \mathrm{~ms}$ in one of four possible positions $\left(\left(10^{\circ}, 10^{\circ}\right),\left(-10^{\circ}, 10^{\circ}\right)\right.$, $630\left(-10^{\circ},-10^{\circ}\right)$ and $\left.\left(10^{\circ},-10^{\circ}\right)\right)$ in a randomly interleaved order. At this time, the monkeys had to 631 release the bar within 150-800 ms after target onset. Success conditioned reward delivery. (3) Memory-guided saccade Task (figure 1B.3): A red fixation cross $\left(0.7 \mathrm{x} 0.7^{\circ}\right)$ appeared in the center of the screen and the monkeys were required to hold fixation for $500 \mathrm{msec}$, within a fixation window of $1.5 \times 1.5^{\circ}$. A squared green cue $\left(0.28 \times 0.28^{\circ}\right)$ was then flashed for $100 \mathrm{~ms}$ at one of four possible locations $\left(\left(10^{\circ}, 10^{\circ}\right),\left(-10^{\circ}, 10^{\circ}\right),\left(-10^{\circ},-10^{\circ}\right)\right.$ and $\left.\left(10^{\circ},-10^{\circ}\right)\right)$. The monkeys had to continue maintain fixation on the central fixation point for another 700-1900 ms until the fixation point disappeared. The monkeys were then required to make a saccade towards the memorized location of the cue within $500-800 \mathrm{~ms}$ from fixation point 639 disappearance, and a spatial tolerance of $4^{\circ} \times 4^{\circ}$. On success, a target, identical to the cue was presented at the cued location and the monkeys were required to fixate it and detect a change in its color by a bar release within 150-800 ms from color change. Success in all of these successive requirements conditioned reward delivery.

\section{Neural recordings:}


On each session, bilateral simultaneous recordings in the two FEFs were carried out using two 24- contact Plexon U-probes. The contacts had an interspacing distance of $250 \mu \mathrm{m}$.

646 Neural data was acquired with the Plexon Omniplex ${ }^{\circledR}$ neuronal data acquisition system. The 647 data was amplified 400 times and digitized at 40,000 Hz. The MUA neuronal data was high648 pass filtered at $300 \mathrm{~Hz}$. The LFP neuronal data was filtered between 0.5 and $300 \mathrm{~Hz}$. In the 649 present paper, all analyses are performed on the multi-unit activity recorded on each of the 48 650 recording contacts. A threshold defining the multi-unit activity was applied independently for 651 each recording contact and before the actual task-related recordings started. All further 652 analyses of the data were performed in Matlab ${ }^{\mathrm{TM}}$ and using FieldTrip ${ }^{88}$ and the Wavelet 653 Coherence Matlab Toolbox ${ }^{89}$, both open source Matlab ${ }^{\mathrm{TM}}$ toolboxes.

\section{Data Analysis}

Data preprocessing. Overall, MUA recordings were collected from 48 recording channels on 26 independent recording sessions (13 for M1 and 13 for M2). We excluded from subsequent analyses all channels with less than 5 spikes per seconds. For each session, we identified the task-related channels based on a statistical change (one-way ANOVA, $\mathrm{p}<0.05$ ) in the MUA neuronal activity in the memory-guided saccade task, in response to either cue presentation ([0 400] $\mathrm{ms}$ after cue onset) against a pre-cue baseline ([-100 0] ms relative to cue onset), or to saccade execution go signal and to saccade execution (i.e. fixation point off, [0 400] ms after go signal) against a pre-go signal baseline ([-100 0] ms relative to go signal), irrespective of the spatial configuration of the trial. In total, 671 channels were retained for further analyses out of 1248 channels.

Distance between recording sites. For each electrode, pairs of MUA recordings were classified along four possible distance categories: D1, spacing of $250 \mu \mathrm{m}$; D2, spacing of 500 $\mu \mathrm{m}$; D3, spacing of $750 \mu \mathrm{m}$ and $\mathrm{D} 4$, spacing of $1 \mathrm{~mm}$. These distances are an indirect proxy to actual cortical distance, as the recordings were performed tangentially to cortical surface, i.e. more or less parallel to sulcal surface.

MUA spatial selectivity. FEF neurons are characterized by a strong visual, saccadic, spatial memory and spatial attention selectivity ${ }^{30,42,43}$. We used a one-way ANOVA $(\mathrm{p}<0.05)$ to identify the spatially selective channels in response to cue presentation $\left(\left[\begin{array}{ll}0 & 400\end{array}\right] \mathrm{ms}\right.$ following cue onset) and to the saccade execution go signal ([0 400] ms following go signal). 
676 modulations were considered as preferred and negative modulations as least preferred. For 677 example, in a given session, the MUA signal recorded on channel 1 of a probe placed in the 678 left FEF, could have as best preferred position p1 the upper right quadrant, the next best preferred position $\mathrm{p} 2$ the lower right quadrant, the next preferred position $\mathrm{p} 3$ the upper left quadrant and the least preferred position $\mathrm{p} 4$ the lower left quadrant. The MUA signal recorded on channel 14 of this same probe, could have as best preferred position $\mathrm{p} 1$ the lower right quadrant, the next best preferred position p2 the upper right quadrant, the next preferred position $\mathrm{p} 3$ the lower left quadrant and the least preferred position $\mathrm{p} 4$ the upper left quadrant. Positions with no significant modulation in any task epoch were labeled as p0 (no selectivity for this position). Once this was done, for each electrode, pairs of MUA recordings were classified along two possible functional categories: pairs with the same spatial selectivity (SSS pairs, sharing the same p1) and pairs with different spatial selectivities (DSS pairs, such that the p1 of one MUA is a p0 for the other MUA). For the sake of clarity, we do not consider partial spatial selectivity pairs (such that the p1 of one MUA is a non-preferred, p2, p3 or $\mathrm{p} 4$ for the other MUA).

MUA layer attribution. As stated above, our recordings are not tangential to cortical surface. As a proxy to attribute a given recording channel to upper or lower cortical layers we proceeded as follows. For each electrode and each channel, we estimated, at the time of cue onset in the memory-guided saccade task (100ms-500ms from cue onset), the spike-field coherence in the alpha range (6 to $16 \mathrm{~Hz}$ ) and the gamma range (40 to $60 \mathrm{~Hz}$ ). Based on previous literature ${ }^{90}$, we used the ratio between the alpha and gamma spike field-coherence as a proxy to assign the considered LFP signals to a deep cortical layer site (high alpha / gamma spike-field coherence ratio) or to a superficial cortical layer site (low alpha / gamma spikefield coherence ratio). We also categorized MUA signals into visual, visuo-motor and motor categories, as in Cohen et al. (2009). Briefly, average firing rates were computed in 3 epochs: [-100 0] ms before cue onset (baseline), [0 200] ms after cue onset (visual), and [0 200] ms before saccade onset (movement). Neurons with activity statistically significantly different from the baseline (Wilcoxon rank-sum test, $P<0.05$ ) after cue onset were categorized as visual. Neurons with activity statistically significantly different from the baseline (Wilcoxon rank-sum test, $P<0.05)$ before saccade onset were categorized as oculomotor. Neurons that were active in both epochs were categorized as visuo-movement neurons. The LFP categorization along the alpha to gamma spike-field coherence ratio strongly coincided with the classification of the MUA signals into purely visual sites (low alpha and gamma spike- 
field coherence ratio, input FEF layers) and visuo-motor sites (high alpha and gamma spikefield coherence ratio, output FEF layers, figure 4).

Noise Correlations. The aim of the present work is to quantify task effects onto the spiking statistics of the FEF spiking activity during equivalent task-fixation epochs. The statistics that we discuss is that of noise correlations between the MUA activities on the different simultaneously recorded signals. For each channel, and each task, intervals of interest of $200 \mathrm{~ms}$ were defined during the fixation epoch from $300 \mathrm{~ms}$ to $500 \mathrm{~ms}$ from eye fixation onset. Specifically, for each channel i, and each trial $k$, the average neuronal response $r_{i}(k)$ for this time interval was calculated and $z$-score normalized into $z_{i}(k)$, where $z_{i}(k)=r_{i}(k)$ $\mu_{\mathrm{i}} / \mathrm{std}_{\mathrm{i}}$ and $\mu_{\mathrm{i}}$ and $\mathrm{std}_{\mathrm{i}}$ respectively correspond to the mean firing rate and standard deviation around this mean during the interval of interest of the channel of interest i. This z-score normalization allows to capture the changes in neuronal response variability independently of changes in mean firing rates. Noise correlations between pairs of MUA signals during the interval of interest were then defined as the Pearson correlation coefficient between the zscored individual trial neuronal responses of each MUA signal over all trials. Only positive significant noise correlations are considered, unless stated otherwise. In any given recording session, noise correlations were calculated between MUA signals recorded from the same electrode, thus specifically targeting intra-cortical correlations. This procedure was applied independently for each task. Depending on the question being asked, noise correlations were either computed on activities aligned on fixation onset, or on activities aligned on target (Fixation and Target detection task) or saccade execution (memory guided saccade task) signals.

In order to control for the fact that the observed changes in noise correlations cannot be attributed to changes in other firing rate metrics, several statistics were also extracted, from comparable task epochs, from 300 to $500 \mathrm{~ms}$ following trial initiation and fixation onset. None of these metrics were significantly affected by the task. Specifically, we analyzed (a) mean firing rate (ANOVA, $>>0.5$ ), (b) the standard error around this mean firing rate (ANOVA, p>0.6), and (c) the corresponding Fano factor (ANOVA, p>0.7). These data, reproducing previous reports ${ }^{54,91}$ are not shown.

Oscillations in noise correlations. To measure oscillatory patterns in the noise correlation time-series data, we computed, for each task, and each session $(\mathrm{N}=12)$, noise correlations over time (over successive $200 \mathrm{~ms}$ intervals, sliding by $10 \mathrm{~ms}$, running from $300 \mathrm{~ms}$ to $1500 \mathrm{~ms}$ following eye fixation onset for Fixation and Target detection tasks and 
742 from $300 \mathrm{~ms}$ to $1500 \mathrm{~ms}$ following cue offset form Memory-guided saccade task). A wavelet

743 transform $^{88}$ was then applied on each session's noise correlation time series. Statistical

744 differences in the noise correlation power frequency spectra were assessed using a non-

745 parametric Friedman test. When computing the noise correlations in time, we equalized the

746 number of trials for all tasks and all conditions so as to prevent any bias that could be

747 introduced by unequal numbers of trials. To control that oscillations in noise correlations in

748 time cannot be attributed to changes in spiking activity, a wavelet analysis was also run onto

749 MUA time series data (data not shown).

750 Spike field Coherence (SFC). In our study monkeys performed three tasks with

751 different task engagement levels. For each selected channel, SFC spectra were calculated

752 between the spiking activity obtained in one channel and the LFP activity from the next

753 adjacent channel in the time interval running from $300 \mathrm{~ms}$ to $1500 \mathrm{~ms}$ following eye fixation

754 onset (Fixation and Target detection task) or cue offset (Memory guided saccade task). We

755 used a single Hanning taper and applied convolution transform to the Hanning-tapered trials.

756 We equalized the number of trials for all tasks so as to prevent any bias that could be

757 introduced by unequal numbers of trials. We used a 4 cycles length per frequency. The

758 memory guided saccade task is known to involve spatial processes during the cue to target

759 interval that bias spike field coherence. In this task, SFC was thus measured separately for

760 trials in which the cued location matched the preferred spatial location of the channel and

761 trials in which the cued location did not match the preferred spatial location of the channel.

762 Statistics were computed across channels x sessions, using a non-parametric Friedman test.

763

764

765

766

767

768

769

770

771

772

773

774

775
Modulation of behavioral performance by phase of noise correlation alpha and beta

rhythmicity. To quantify the effect of noise correlation oscillations onto behavioral performance, we used a complex wavelet transform analysis (Fieldtrip, Oostenveld et al. 2011) to compute, for each session and each task, in the noise correlations, the phase of the frequencies of interest (alpha / beta) following eye fixation onset (for the Fixation and Target detection tasks) or cue offset (for the Memory guided saccade task). For each session, we identified hit and miss trials falling at zero phase of the frequency of interest $(+/-\pi / 140)$ with respect to target presentation or fixation point offset time. In the fixation task, premature fixation aborts by anticipatory manual response or eye fixation failure were considered as misses. Hit rates (HR) were computed for this zero phase bin. We then shifted this phase window by $\pi / 70$ steps and recalculated the HR, repeating this procedure to generate phasedetection HR functions, across all phases, for each frequency of interest ${ }^{92}$. For each session, 
776 the phase bin for which hit rate was maximal was considered as the optimal phase. The effect

777 of a given frequency (alpha or beta) onto behavior corresponds to the difference between HR

778 at this optimal phase and HR at the anti-optimal phase (optimal phase $+\pi$ ). To test for

779 statistical significance, observed hit/miss phases were randomized across trials so as to shuffle

780 the temporal relationship between phases and behavioral performance. This procedure was

781 repeated 1000 times. 95\% CI was then computed and compared to the observed behavioral

782 data.

783

784 


\section{Aknowledgments}

786 S.B.H.H. was supported by ANR grant ANR-14-CE13-0005-1. C.G. was supported by the

787 French Ministère de l'Enseignement Supérieur et de la Recherche. S.B.H. was supported by 788 ANR grant ANR-11-BSV4-0011, ANR grant ANR-14-CE13-0005-1, and the LABEX 789 CORTEX (ANR-11-LABX-0042) of Universite de Lyon, within the program Investissements 790 d'Avenir (ANR-11-IDEX-0007) operated by the French National Research Agency (ANR).

791 E.A. was supported by the CNRS-DGA and Fondation pour la Recherche Médicale. We thank 792 research engineer Serge Pinède for technical support and Jean-Luc Charieau and Fabrice 793 Hérant for animal care. All procedures were approved by the local animal care committee 794 (C2EA42-13-02-0401-01) in compliance with the European Community Council, Directive 795 2010/63/UE on Animal Care.

796

\section{Authors contributions}

798 Conceptualization, S.B.H. S.B.H.H. and C.G.; Methodology, S.B.H., S.B.H.H., C.G., E.A., 799 C.W.; Investigation, S.B.H., S.B.H.H., C.G., E.A. and C.W.; Writing - Original Draft, S.B.H. 800 S.B.H.H. and C.G.; Writing - Review \& Editing, S.B.H. S.B.H.H. and C.G.; Funding 801 Acquisition, S.B.H.; Supervision, S.B.H.

802 
803

804

805

806

807

808

809

810

811

812

813

814

815

816

817

818

819

820

821

822

823

824

825

826

827

828

829

830

831

832

833

834

835

836

837

838

839

840

841

842

843

844

845

846

847

848

849

850

\section{References}

1. Ts'o, D. Y., Gilbert, C. D. \& Wiesel, T. N. Relationships between horizontal interactions and functional architecture in cat striate cortex as revealed by cross-correlation analysis. J. Neurosci. 6, 1160-1170 (1986).

2. Engel, A. K., König, P., Kreiter, A. K. \& Singer, W. Interhemispheric Synchronization of Oscillatory Neuronal Responses in Cat Visual Cortex. Science 252, 1177-1179 (1991).

3. Ahissar, E. et al. Dependence of Cortical Plasticity on Correlated Activity of Single Neurons and on Behavioral Context. Science 257, 1412-1415 (1992).

4. Zohary, E., Shadlen, M. N. \& Newsome, W. T. Correlated neuronal discharge rate and its implications for psychophysical performance. Nature 370, 140-143 (1994).

5. Vaadia, E. et al. Dynamics of neuronal interactions in monkey cortex in relation to behavioural events. Nature 373, 515-518 (1995).

6. Narayanan, N. S. \& Laubach, M. Top-down control of motor cortex ensembles by dorsomedial prefrontal cortex. Neuron 52, 921-931 (2006).

7. Cohen, J. Y. et al. Cooperation and competition among frontal eye field neurons during visual target selection. J. Neurosci. Off. J. Soc. Neurosci. 30, 3227-3238 (2010).

8. Poulet, J. F. A. \& Petersen, C. C. H. Internal brain state regulates membrane potential synchrony in barrel cortex of behaving mice. Nature 454, 881-885 (2008).

9. Stark, E., Globerson, A., Asher, I. \& Abeles, M. Correlations between groups of premotor neurons carry information about prehension. J. Neurosci. Off. J. Soc. Neurosci. 28, 10618-10630 (2008).

10. Abbott, L. F. \& Dayan, P. The Effect of Correlated Variability on the Accuracy of a Population Code. Neural Computation 11, 91-101 (1999).

11. Sompolinsky, H., Yoon, H., Kang, K. \& Shamir, M. Population coding in neuronal systems with correlated noise. Phys. Rev. E Stat. Nonlin. Soft Matter Phys. 64, 051904 (2001).

12. Averbeck, B. B., Latham, P. E. \& Pouget, A. Neural correlations, population coding and computation. Nat. Rev. Neurosci. 7, 358-366 (2006).

13. Ecker, A. S., Berens, P., Tolias, A. S. \& Bethge, M. The effect of noise correlations in populations of diversely tuned neurons. J. Neurosci. Off. J. Soc. Neurosci. 31, 1427214283 (2011).

14. Moreno-Bote, R. et al. Information-limiting correlations. Nat. Neurosci. 17, 1410 (2014).

15. Ekstrom, L. B., Roelfsema, P. R., Arsenault, J. T., Bonmassar, G. \& Vanduffel, W. Bottom-up dependent gating of frontal signals in early visual cortex. Science 321, 414417 (2008).

16. Shadlen, M. N. \& Newsome, W. T. The variable discharge of cortical neurons: implications for connectivity, computation, and information coding. J. Neurosci. Off. J. Soc. Neurosci. 18, 3870-3896 (1998).

17. Ecker, A. S. et al. State Dependence of Noise Correlations in Macaque Primary Visual Cortex. Neuron 82, 235-248 (2014).

18. Goris, R. L. T., Movshon, J. A. \& Simoncelli, E. P. Partitioning neuronal variability. Nat. Neurosci. 17, 858-865 (2014).

19. Wimmer, K. et al. Sensory integration dynamics in a hierarchical network explains choice probabilities in cortical area MT. Nat. Commun. 6, 6177 (2015).

20. Ben-Yishai, R., Bar-Or, R. L. \& Sompolinsky, H. Theory of orientation tuning in visual cortex. Proc. Natl. Acad. Sci. U. S. A. 92, 3844-3848 (1995).

21. Litwin-Kumar, A. \& Doiron, B. Slow dynamics and high variability in balanced cortical networks with clustered connections. Nat. Neurosci. 15, 1498-1505 (2012). 
22. Ly, C., Middleton, J. W. \& Doiron, B. Cellular and circuit mechanisms maintain low spike co-variability and enhance population coding in somatosensory cortex. Front. Comput. Neurosci. 6, 7 (2012).

23. Kanitscheider, I., Coen-Cagli, R. \& Pouget, A. Origin of information-limiting noise correlations. Proc. Natl. Acad. Sci. U. S. A. 112, E6973-6982 (2015).

24. Cohen, M. R. \& Maunsell, J. H. R. Attention improves performance primarily by reducing interneuronal correlations. Nat. Neurosci. 12, 1594-1600 (2009).

25. Meyers, E. M., Qi, X.-L. \& Constantinidis, C. Incorporation of new information into prefrontal cortical activity after learning working memory tasks. Proc. Natl. Acad. Sci. U. S. A. 109, 4651-4656 (2012).

26. $\mathrm{Gu}, \mathrm{Y}$. et al. Perceptual learning reduces interneuronal correlations in macaque visual cortex. Neuron 71, 750-761 (2011).

27. Ni, A. M., Ruff, D. A., Alberts, J. J., Symmonds, J. \& Cohen, M. R. Learning and attention reveal a general relationship between population activity and behavior. Science 359, 463-465 (2018).

28. Buschman, T. J. \& Miller, E. K. Top-down versus bottom-up control of attention in the prefrontal and posterior parietal cortices. Science 315, 1860-1862 (2007).

29. Wardak, C., Ibos, G., Duhamel, J.-R. \& Olivier, E. Contribution of the monkey frontal eye field to covert visual attention. J. Neurosci. Off. J. Soc. Neurosci. 26, 4228-4235 (2006).

30. Ibos, G., Duhamel, J.-R. \& Ben Hamed, S. A functional hierarchy within the parietofrontal network in stimulus selection and attention control. J. Neurosci. Off. J. Soc. Neurosci. 33, 8359-8369 (2013).

31. Gregoriou, G. G., Gotts, S. J., Zhou, H. \& Desimone, R. High-frequency, long-range coupling between prefrontal and visual cortex during attention. Science 324, 1207-1210 (2009).

32. Gregoriou, G. G., Gotts, S. J. \& Desimone, R. Cell-type-specific synchronization of neural activity in FEF with V4 during attention. Neuron 73, 581-594 (2012).

33. Armstrong, K. M., Chang, M. H. \& Moore, T. Selection and maintenance of spatial information by frontal eye field neurons. J. Neurosci. Off. J. Soc. Neurosci. 29, 1562115629 (2009).

34. Schölvinck, M. L., Saleem, A. B., Benucci, A., Harris, K. D. \& Carandini, M. Cortical state determines global variability and correlations in visual cortex. J. Neurosci. Off. J. Soc. Neurosci. 35, 170-178 (2015).

35. Arieli, A., Sterkin, A., Grinvald, A. \& Aertsen, A. Dynamics of ongoing activity: explanation of the large variability in evoked cortical responses. Science 273, 1868-1871 (1996).

36. Bair, W., Zohary, E. \& Newsome, W. T. Correlated firing in macaque visual area MT: time scales and relationship to behavior. J. Neurosci. Off. J. Soc. Neurosci. 21, 1676-1697 (2001).

37. Bryant, H. L., Marcos, A. R. \& Segundo, J. P. Correlations of neuronal spike discharges produced by monosynaptic connections and by common inputs. J. Neurophysiol. 36, 205225 (1973).

38. Constantinidis, C. \& Goldman-Rakic, P. S. Correlated discharges among putative pyramidal neurons and interneurons in the primate prefrontal cortex. J. Neurophysiol. 88, 3487-3497 (2002).

39. Lee, D., Port, N. L., Kruse, W., Georgopoulos, A. P. \& Neurology. Variability and correlated noise in the discharge of neurons in motor and parietal areas of the primate cortex. J Neurosci 18,1161-1170 (1998). 
40. Smith, M. A. \& Kohn, A. Spatial and Temporal Scales of Neuronal Correlation in Primary Visual Cortex. J. Neurosci. 28, 12591-12603 (2008).

41. Mohler, C. W., Goldberg, M. E. \& Wurtz, R. H. Visual receptive fields of frontal eye field neurons. Brain Res. 61, 385-389 (1973).

42. Bruce, C. J. \& Goldberg, M. E. Primate frontal eye fields: I. Single neurons discharging before saccades. J. Neurophysiol. 53, 603-635 (1985).

43. Astrand, E., Ibos, G., Duhamel, J.-R. \& Ben Hamed, S. Differential dynamics of spatial attention, position, and color coding within the parietofrontal network. J. Neurosci. Off. J. Soc. Neurosci. 35, 3174-3189 (2015).

44. Segraves, M. A. \& Goldberg, M. E. Functional properties of corticotectal neurons in the monkey's frontal eye field. J. Neurophysiol. 58, 1387-1419 (1987).

45. Schall, J. D. Neuronal activity related to visually guided saccades in the frontal eye fields of rhesus monkeys: comparison with supplementary eye fields. J. Neurophysiol. 66, 559579 (1991).

46. Schall, J. D. \& Hanes, D. P. Neural basis of saccade target selection in frontal eye field during visual search. Nature 366, 467-469 (1993).

47. Schall, J. D., Hanes, D. P., Thompson, K. G. \& King, D. J. Saccade target selection in frontal eye field of macaque. I. Visual and premovement activation. J. Neurosci. Off. $J$. Soc. Neurosci. 15, 6905-6918 (1995).

48. Schall, J. D. \& Thompson, K. G. Neural selection and control of visually guided eye movements. Annu. Rev. Neurosci. 22, 241-259 (1999).

49. Buffalo, E. A., Fries, P., Landman, R., Buschman, T. J. \& Desimone, R. Laminar differences in gamma and alpha coherence in the ventral stream. Proc. Natl. Acad. Sci. U. S. A. 108, 11262-11267 (2011).

50. Womelsdorf, T., Fries, P., Mitra, P. P. \& Desimone, R. Gamma-band synchronization in visual cortex predicts speed of change detection. Nature 439, 733-736 (2006).

51. Womelsdorf, T., Anton-Erxleben, K. \& Treue, S. Receptive field shift and shrinkage in macaque middle temporal area through attentional gain modulation. J. Neurosci. Off. $J$. Soc. Neurosci. 28, 8934-8944 (2008).

52. Anton-Erxleben, K., Henrich, C. \& Treue, S. Attention changes perceived size of moving visual patterns. J. Vis. 7, 5.1-9 (2007).

53. Ben Hamed, S., Duhamel, J.-R., Bremmer, F. \& Graf, W. Visual receptive field modulation in the lateral intraparietal area during attentive fixation and free gaze. Cereb. Cortex N. Y. N 1991 12, 234-245 (2002).

54. Astrand, E., Wardak, C., Baraduc, P. \& Ben Hamed, S. Direct Two-Dimensional Access to the Spatial Location of Covert Attention in Macaque Prefrontal Cortex. Curr. Biol. CB 26, 1699-1704 (2016).

55. Engel, A. K. \& Fries, P. Beta-band oscillations--signalling the status quo? Curr. Opin. Neurobiol. 20, 156-165 (2010).

56. Thut, G., Nietzel, A., Brandt, S. A. \& Pascual-Leone, A. Alpha-band electroencephalographic activity over occipital cortex indexes visuospatial attention bias and predicts visual target detection. J. Neurosci. Off. J. Soc. Neurosci. 26, 9494-9502 (2006).

57. Rihs, T. A., Michel, C. M. \& Thut, G. A bias for posterior alpha-band power suppression versus enhancement during shifting versus maintenance of spatial attention. NeuroImage 44, 190-199 (2009).

58. Varela, F. J., Toro, A., John, E. R. \& Schwartz, E. L. Perceptual framing and cortical alpha rhythm. Neuropsychologia 19, 675-686 (1981).

59. Mathewson, K. E., Gratton, G., Fabiani, M., Beck, D. M. \& Ro, T. To See or Not to See: Prestimulus $\alpha$ Phase Predicts Visual Awareness. J. Neurosci. 29, 2725-2732 (2009). 
60. Busch, N. A. \& VanRullen, R. Spontaneous EEG oscillations reveal periodic sampling of visual attention. Proc. Natl. Acad. Sci. U. S. A. 107, 16048-16053 (2010).

61. Klimesch, W. EEG-alpha rhythms and memory processes. Int. J. Psychophysiol. Off. J. Int. Organ. Psychophysiol. 26, 319-340 (1997).

62. Constantinidis, C. \& Klingberg, T. The neuroscience of working memory capacity and training. Nat. Rev. Neurosci. 17, 438-449 (2016).

63. Gutnisky, D. A. \& Dragoi, V. Adaptive coding of visual information in neural populations. Nature 452, 220-224 (2008).

64. Poort, J. \& Roelfsema, P. R. Noise correlations have little influence on the coding of selective attention in area V1. Cereb. Cortex N. Y. N 1991 19, 543-553 (2009).

65. Reich, D. S. Independent and Redundant Information in Nearby Cortical Neurons. Science 294, 2566-2568 (2001).

66. Mitchell, J. F., Sundberg, K. A. \& Reynolds, J. H. Spatial attention decorrelates intrinsic activity fluctuations in macaque area V4. Neuron 63, 879-888 (2009).

67. Gawne, T. J., Kjaer, T. W., Hertz, J. A. \& Richmond, B. J. Adjacent visual cortical complex cells share about $20 \%$ of their stimulus-related information. Cereb. Cortex N. Y. N 1991 6, 482-489 (1996).

68. Gawne, T. J. \& Richmond, B. J. How independent are the messages carried by adjacent inferior temporal cortical neurons? J. Neurosci. Off. J. Soc. Neurosci. 13, 2758-2771 (1993).

69. Cohen, M. R. \& Newsome, W. T. Context-dependent changes in functional circuitry in visual area MT. Neuron 60, 162-173 (2008).

70. Huang, X. \& Lisberger, S. G. Noise Correlations in Cortical Area MT and Their Potential Impact on Trial-by-Trial Variation in the Direction and Speed of Smooth-Pursuit Eye Movements. J. Neurophysiol. 101, 3012-3030 (2009).

71. Ecker, A. S. et al. Decorrelated Neuronal Firing in Cortical Microcircuits. Science 327, 584-587 (2010).

72. Renart, A. et al. The asynchronous state in cortical circuits. Science 327, 587-590 (2010).

73. Chandrasekaran, C. Computational principles and models of multisensory integration. Curr. Opin. Neurobiol. 43, 25-34 (2017).

74. Constantinidis, C. \& Goldman-Rakic, P. S. Correlated discharges among putative pyramidal neurons and interneurons in the primate prefrontal cortex. J. Neurophysiol. 88, 3487-3497 (2002).

75. Lee, D., Port, N. L., Kruse, W., Georgopoulos, A. P. \& Neurology. Variability and correlated noise in the discharge of neurons in motor and parietal areas of the primate cortex. J Neurosci 18, 1161-1170. (1998).

76. Seriès, P., Latham, P. E. \& Pouget, A. Tuning curve sharpening for orientation selectivity: coding efficiency and the impact of correlations. Nat. Neurosci. 7, 1129-1135 (2004).

77. Desimone, R. \& Duncan, J. Neural Mechanisms of Selective Visual Attention. Annu. Rev. Neurosci. 18, 193-222 (1995).

78. Chalk, M. et al. Attention Reduces Stimulus-Driven Gamma Frequency Oscillations and Spike Field Coherence in V1. Neuron 66, 114 (2010).

79. Engel, A. K., Fries, P. \& Singer, W. Dynamic predictions: oscillations and synchrony in top-down processing. Nat. Rev. Neurosci. 2, 704-716 (2001).

80. Okazaki, M., Kaneko, Y., Yumoto, M. \& Arima, K. Perceptual change in response to a bistable picture increases neuromagnetic beta-band activities. Neurosci. Res. 61, 319-328 (2008).

81. Iversen, J. R., Repp, B. H. \& Patel, A. D. Top-down control of rhythm perception modulates early auditory responses. Ann. N. Y. Acad. Sci. 1169, 58-73 (2009). 
82. Buschman, T. J. \& Miller, E. K. Serial, covert shifts of attention during visual search are reflected by the frontal eye fields and correlated with population oscillations. Neuron 63 , 386-396 (2009).

83. Joundi, R. A., Jenkinson, N., Brittain, J.-S., Aziz, T. Z. \& Brown, P. Driving oscillatory activity in the human cortex enhances motor performance. Curr. Biol. 22, 403-407 (2012).

84. Lalo, E. et al. Phasic increases in cortical beta activity are associated with alterations in sensory processing in the human. Exp. Brain Res. 177, 137-145 (2007).

85. Courtemanche, R., Fujii, N. \& Graybiel, A. M. Synchronous, focally modulated beta-band oscillations characterize local field potential activity in the striatum of awake behaving monkeys. J. Neurosci. Off. J. Soc. Neurosci. 23, 11741-11752 (2003).

86. Fiebelkorn, I. C., Pinsk, M. A. \& Kastner, S. A Dynamic Interplay within the Frontoparietal Network Underlies Rhythmic Spatial Attention. Neuron 99, 842-853.e8 (2018).

87. Fiebelkorn, I. C. \& Kastner, S. A Rhythmic Theory of Attention. Trends Cogn. Sci. 23, 87-101 (2018).

88. Oostenveld, R., Fries, P., Maris, E. \& Schoffelen, J.-M. FieldTrip: Open Source Software for Advanced Analysis of MEG, EEG, and Invasive Electrophysiological Data. Intell Neurosci. 2011, 1:1-1:9 (2011).

89. Grinsted, A., Moore, J. C. \& Jevrejeva, S. Application of the cross wavelet transform and wavelet coherence to geophysical time series. Nonlinear Process. Geophys. 11, 561-566 (2004).

90. Buffalo, E. A., Fries, P., Landman, R., Buschman, T. J. \& Desimone, R. Laminar differences in gamma and alpha coherence in the ventral stream. Proc. Natl. Acad. Sci. U. S. A. 108, 11262-11267 (2011).

91. Cohen, M. R. \& Maunsell, J. H. R. Attention improves performance primarily by reducing interneuronal correlations. Nat. Neurosci. 12, 1594-1600 (2009).

92. Fiebelkorn, I. C. et al. Cortical cross-frequency coupling predicts perceptual outcomes. NeuroImage 69, 126-137 (2013). 